\title{
Glucosinolate Content and Myrosinase Activity in Rapid-cycling Brassica oleracea Grown in a Controlled Environment
}

\author{
Craig S. Charron and Carl E. Sams ${ }^{1}$ \\ Department of Plant Sciences, The University of Tennessee, Knoxville, TN 37996
}

ADDITIONAL INDEX WORDS. isothiocyanate, glucoraphanin, sulforaphane, anticarcinogen, thioglucosidase, biofumigation

\begin{abstract}
Crops of the Brassicaceae contain glucosinolates(GSs), which when hydrolyzed by the enzyme myrosinase, generate products involved in cancer chemoprotection, plant defense, and plant-insect interactions. A rapid-cycling base population of $B$. oleracea $\mathrm{L}$. was grown in a hydroponic system in a controlled environment to determine the roles of temperature, photosynthetic photon flux $(P P F)$, and photoperiod in GS concentration and myrosinase activity. The concentration of total GSs in leaves was $44 \%$ and $114 \%$ higher at 12 and $32{ }^{\circ} \mathrm{C}$ respectively than at $22{ }^{\circ} \mathrm{C}$ under constant light of $300 \mu \mathrm{mol} \cdot \mathrm{m}^{-2} \cdot \mathrm{s}^{-1}$. The concentration of glucoraphanin, the precursor to sulforaphane, a compound with chemoprotective properties, was 5 -fold higher at 32 than at $22^{\circ} \mathrm{C}$. Total GSs were $\approx 50 \%$ lower in roots at $12{ }^{\circ} \mathrm{C}$ and 32 than at $22{ }^{\circ} \mathrm{C}$. Total GSs in leaves decreased $20 \%$ when $P P F$ was increased from 200 to $400 \mu \mathrm{mol} \cdot \mathrm{m}^{-2} \cdot \mathrm{s}^{-1}$. $\mathrm{Myrosinase}^{-}$ activity on a fresh weight basis (activity-FW) was $\approx 30 \%$ higher in leaves and stems at 12 and $32{ }^{\circ} \mathrm{C}$ than at $22{ }^{\circ} \mathrm{C}$, and $\approx 30 \%$ higher in leaves grown at 200 and $400 \mu \mathrm{mol} \cdot \mathrm{m}^{-2} \cdot \mathrm{s}^{-1}$ than at $300 \mu \mathrm{mol} \cdot \mathrm{m}^{-2} \cdot \mathrm{s}^{-1}$. Consideration of climatic factors that influence the glucosinolate-myrosinase system may be necessary to optimize the planting and cultivation of $B$ rassica crops for maximum health benefits.
\end{abstract}

Glucosinolates (GSs) are secondary plant metabolites which occur in sixteen families of dicotyledonous angiosperms. Due primarily to the bioactivity of their hydrolysis products, GSs are credited with fungicidal, bactericidal, nematocidal, and allelopathic activity (Fahey et al., 2001). Catabolism of GSs by the enzyme myrosinase (EC 3.2.3.1) produces degradation products such as isothiocyanates, thiocyanates, nitriles, epithionitriles, and oxazolidine-2-thiones (Rosa et al., 1997).

Recent research has shown that GS degradation products have chemoprotective properties (Gamet-Payrastre et al., 2000; Zhang et al., 1994). Since vegetables of the Brassicaceae contain GSs and since interest in health matters has broadened substantially, this research has increased rapidly in scope and depth. Consumption of cruciferous vegetables has been linked with decreased incidence of several cancers (London, 2000; Michaud et al., 1999; Talalay, 1999). The proposed chemopreventive mechanisms triggered by GS degradation products involve the induction of mammalian Phase 2 detoxication enzymes (such as quinone reductase and glutathione transferase) and the inhibition of carcinogen-activating Phase 1 enzymes [such as the cytochrome P-450s (Fahey et al., 1997; Talalay, 1999; Yang et al., 1994)]. Sulforaphane, the isothiocyanate derivative of glucoraphanin (4-methylsulfinylbutyl GS; GR), is a particularly effective inducer of Phase 2 enzymes (Fahey and Talalay, 1999). The isothiocyanate derivatives of glucoiberin and glucoerucin also induce phase 2 enzymes but are about $10 \%$ as effective as sulforaphane (Zhang et al., 1992).

The potential health benefits of GS-containing crops amplify the importance of illuminating factors that influence the GS-myrosinase system. Environmental factors such as temperature and photosynthetic photon flux $(P P F)$ significantly impact plant growth and development and thus may affect GS content or myrosinase activity. A number of studies have determined that glucosinolate (GS) concentrations in Brassica crops vary by growing season. During the fall and winter when temperatures and light intensity

Received for publication 22 July 2003. Accepted for publication 21 Oct. 2003. 1To whom reprint requests should be addressed; e-mail carlsams@utk.edu. are lower, days are shorter, and water availability is higher, glucosinolate concentrations tend to be lower (Sang et al., 1986; Sarwar and Kirkegaard, 1998). Glucosinolate concentrations in several cruciferous vegetables were higher during a year characterized by high average temperature and low average 10-d rainfall compared to the same vegetables grown in a year with lower average temperature and higher average 10-d rainfall (Ciska et al., 2000). Total and individual GS concentrations were generally higher in eleven broccoli [B. oleracea L. (Botrytis Group)] cultivars grown during a summer season compared to those grown during a winter season (Rosa and Rodrigues, 2001). None of these studies demonstrated a statistical relationship between GS concentrations and particular climatic conditions.

Controlled environment studies indicated that glucosinolate concentrations in cabbage [B. oleracea L. (Capitata Group)] seedlings varied throughout a 24 -h period and were highest during the dark cycle (Rosa, 1997). A similar study showed that even at constant light, temperature, and relative humidity, GS concentrations varied in cabbage seedlings depending on time of sampling. Moreover, these ultradian variations were more pronounced at 30 than at $20^{\circ} \mathrm{C}$ (Rosa, 1998).

Investigation of how environmental factors during plant growth affect myrosinase activity has been limited. Bones and Iversen (1985) examined Brassica seeds allowed to germinate in darkness, continuous light, or in a 12-h light cycle. They concluded that myrosin cells occurred more frequently in light-grown hypocotyls and roots than in dark-grown plants. Myrosinase from roots of dark-grown Sinapsis alba L. seedlings had higher activity than that from light-grown seedlings, whereas in hypocotyls, no effect of light was evident (Pihakaski and Pihakaski, 1978).

In the wild, myrosinase activity may be important in plant defense, although at a cost to the plant. Increased myrosinase activity in selections of wild $B$. rapa $\mathrm{L}$. was associated with increased resistance to flea beetles but resulted in lower fecundity, perhaps due to production costs of myrosinase or associated defensive pathways (Mitchell-Olds et al., 1996). Li et al. (2000) showed that high levels of myrosinase activity reduced 
the feeding time and area damaged on B. juncea L. by Plutella xylostella. Thus it is plausible that myrosinase activity would be affected by environmental influences that would jeopardize plant health and survival.

The objective of this study was to determine how temperature, $P P F$, and photoperiod affect GS concentrations and myrosinase activity in leaves, stems, and roots of a rapid-cycling base population (RCBP; genome Ccc) of B. oleracea grown in a controlled environment.

\section{Materials and Methods}

Growth ChAMBER CONDITIONS. Plants of an RCBP of B. oleracea (Crucifer Genetics Cooperative, Department of Plant Pathology, University of Wisconsin, Madison, Wis.) were grown in a hydroponic system in a model E 30B Percival Scientific growth chamber (Boone, Iowa). Four plastic nutrient reservoirs, each representing a replication consisting of eight plants (two rows separated by $8 \mathrm{~cm}$, four plants per row, $5 \mathrm{~cm}$ between plants within a row), held $1.8 \mathrm{~L}$ of modified half-strength Hoagland solution. The $\mathrm{pH}$ was adjusted to between 5.8 and 6.2 by adding $2.5 \%$ nitric acid as needed. The nutrient solution was aerated by pumping air through an aeration stone placed in each reservoir.

At each plant location, a plant support medium fashioned from low-density polyurethane foam growing cubes (Smithers-Oasis, Kent, Ohio) was placed so that the support protruded into the nutrient solution. One or two seeds were sown in each support medium. From planting until the first appearance of true leaves, the temperature was $22^{\circ} \mathrm{C}$ and there was constant $P P F$ of 300 $\mu \mathrm{mol} \cdot \mathrm{m}^{-2} \cdot \mathrm{s}^{-1}$.

Treatments. A single environmental parameter was varied in the temperature, $P P F$, and photoperiod experiments (Table 1). Additionally, an experiment was designed to simulate the changing environmental conditions of crops grown in a fall or spring season (Table 2). High and low temperatures were similar to the 10-year monthly averages recorded at the Knoxville Experiment Station, Knoxville, Tenn. Since RCBP plants can grow under constant light, photoperiod was extended beyond that typical of the Southeast. Relative humidity was maintained between $75 \%$ and $95 \%$ by pans of water placed on the bottom of the growth chamber.

Collection AND ANALYSIS OF PLANT MATERIAL. Plants were harvested just before anthesis. Fresh weights were recorded after separation of leaves, stems and roots. Plant tissue was frozen in liquid $\mathrm{N}$, ground into a fine powder in a room at $-3^{\circ} \mathrm{C}$, and stored at $-80{ }^{\circ} \mathrm{C}$ until analyzed.

GLuCOSINOLATE ANALYSIS. A 200-mg lyophilized sample was combined with $1 \mathrm{~mL}$ benzyl GS solution $(1 \mathrm{~mm})$ as an internal standard, $2.0 \mathrm{~mL}$ methanol, and $0.3 \mathrm{~mL}$ barium-lead acetate $(0.6$ M) in a $16 \times 100-\mathrm{mm}$ culture tube and shaken at $60 \mathrm{rpm}$ for $1 \mathrm{~h}$. Each tube was centrifuged at $2000 g_{\mathrm{n}}$ for $10 \mathrm{~min}$. An aliquot of $0.5 \mathrm{~mL}$ supernatant was added to a $1-\mathrm{mL}$ column containing 0.3 $\mathrm{mL}$ DEAE Sephadex A-25 and desulfated by the procedure of Raney and McGregor (1990).

Extracted desulfoglucosinolates were separated with a HewlettPackard (PaloAlto, Calif.) high performance liquid chromatograph (HPLC) using a C-18 ODS reverse-phase column $(250 \times 4.6 \mathrm{~mm}$ ID, $5-\mu \mathrm{m}$ ) and UV detector at a wavelength of $230 \mathrm{~nm}$. The column temperature was set at $35^{\circ} \mathrm{C}$. A flow rate of $1.5 \mathrm{~mL} \cdot \mathrm{min}^{-1}$ was used. The solvent was $100 \%$ water for $1 \mathrm{~min}$. After a 15 -min linear gradient to $75 \%$ water and $25 \%$ acetonitrile, solvent levels were held constant for $5 \mathrm{~min}$, and over the final $5 \mathrm{~min}$ a linear gradient to $100 \%$ water was used. Desulfoglucosinolates were identified and quantified by comparison with authentic standards and previously reported results (Hansen et al., 1995; Kushad et al., 1999).

Myrosinase anAlysis. Each of two $300-\mathrm{mg}$ portions from each sample of frozen plant material was combined with $1 \mathrm{~mL}$ $30 \mathrm{mM}$ citrate phosphate buffer ( $\mathrm{pH} 7.0$ ) containing $1 \mathrm{mM}$ EDTA and ground for $30 \mathrm{~s}$ in a $1.5-\mathrm{mL}$ polyallomer microcentrifuge tube (Beckman Instruments, Palo Alto, Calif.). After centrifugation (model J2-MC; Beckman Instruments, Palo Alto, Calif.) at $30,100 g_{\mathrm{n}}$ and $4{ }^{\circ} \mathrm{C}$ for $4 \mathrm{~min}$, the supernatants from the two tubes were combined.

Myrosinase activity was determined by the decrease in absorbance at $227 \mathrm{~nm}$ measured on a UV160U spectrophotometer (Shimadzu, Kyoto, Japan) resulting from the enzymatic hydrolysis of sinigrin (Palmieri et al., 1982). A 1.5-mL reaction mixture (25 ${ }^{\circ} \mathrm{C}$ ) was prepared that contained $50 \mu \mathrm{L}$ crude extract, $30 \mathrm{~mm}$ citrate phosphate buffer ( $\mathrm{pH} 4.5,5.0,5.5,6.0$, or 6.5), $2.5 \mathrm{~mm}$ sinigrin, and $1 \mathrm{~mm}$ EDTA. Tests for treatment differences in myrosinase activity were applied to the maximum activity measured in samples in the $\mathrm{pH}$ range 4.5 to 6.5 .

In preliminary experiments, it was determined that substrate saturation was achieved with $2.5 \mathrm{~mm}$ sinigrin. The mixture was vortexed for $5 \mathrm{~s}$ in a $13 \times 100-\mathrm{mm}$ polypropylene culture tube (Fisher Scientific, Pittsburgh, Pa.) and transferred to a $1.5 \mathrm{~mL}$ semi-micro cuvette (BrandTech Scientific, Essex, Conn.). A reference mixture was used which did not contain sinigrin. After 1 min, absorbance was measured over a period of $5 \mathrm{~min}$. Activity was determined from the linear slope representing the disappearance of sinigrin from the reaction mixture. A molar extinction coefficient of $6780 \mathrm{M}^{-1} \cdot \mathrm{cm}^{-1}$ was used (Palmieri et al., 1982). A unit (U) of activity was defined as the disappearance of $1 \mu \mathrm{mol}$ sinigrin per min. Thus, to calculate activity from Beer's law: Absorbance $=\varepsilon \times$ pathlength $\times$ concentration and activity (U) - $\left(\Delta\right.$ absorbance $\left.\times \mathrm{min}^{-1}\right) /\left(6780 \mathrm{M}^{-1}\right)$, where $\varepsilon=6780 \mathrm{M}^{-1} \cdot \mathrm{cm}^{-1}$ and pathlength $=1 \mathrm{~cm}$

Protein content of the crude extracts was determined by the Bio-Rad (Bio-Rad Laboratories, Hercules, Calif.) method which is based on the work of Bradford (1976). Bovine serum albumin standard was used to construct a standard curve from which protein concentrations in the crude extracts were calculated and used in the determination of specific activity ( $\mathrm{U} / \mathrm{mg}$ protein).

Statistics. For the temperature, $P P F$, and photoperiod experiments, the experimental design was a completely randomized design with four replications and three levels for each treatment. The mixed procedure (SAS, 1996) was used to determine significant effects based on linear and quadratic contrasts. The fall and spring simulation experiments were compared by $t$ tests. All experiments were repeated. Results from the second experiment were similar to those of the first, and data were therefore pooled and analyzed as a single data set.

\section{Results}

GLucosinolates From Plants gROWN AT DIFFERENT TEMPERATURES. Leaves contained the aliphatic GSs glucoiberin (3-methylsulfinylpropyl GS; GI), gluconapin (3-butenyl GS; GNP), glucoraphanin (4-methylsulfinylbutyl GS; GR), progoitrin (2-hydroxybut-3-enyl GS; PRO), sinigrin (2-propenyl GS; SN), and the indole GSs 4-hydroxyglucobrassicin (4-hydroxyindol3-ylmethyl GS; 4OHGB), glucobrassicin (indol-3-ylmethyl GS; GB), 4-methoxyglucobrassicin (4-methoxyindol-3-ylmethyl GS; 4MGB), and neoglucobrassicin [(1-methoxyindol-3-ylmethyl GS; NGB); Table 3]. Stems contained the aromatic GS gluconasturtiin 
Table 1. Levels of temperature, photosynthetic photon flux $(P P F)$, and photoperiod used in growth chamber experiments.

\begin{tabular}{lccc}
\hline & \multicolumn{3}{c}{ Environmental parameter } \\
\cline { 2 - 4 } Experiment & $\begin{array}{c}\text { Temp } \\
\left({ }^{\circ} \mathrm{C}\right)\end{array}$ & $\begin{array}{c}P P F \\
\left(\mu \mathrm{mol} \cdot \mathrm{m}^{-2} \cdot \mathrm{s}^{-1}\right)\end{array}$ & $\begin{array}{c}\text { Photoperiod } \\
(\mathrm{h})\end{array}$ \\
\hline Temperature & 32 & 300 & 24 \\
Temperature & 22 & 300 & 24 \\
Temperature & 12 & 300 & 24 \\
$P P F$ & 22 & 400 & 24 \\
$P P F$ & 22 & 300 & 24 \\
$P P F$ & 22 & 200 & 24 \\
Photoperiod & 22 & 300 & 24 \\
Photoperiod & 22 & 300 & 18 \\
Photoperiod & 22 & 300 & 12 \\
\hline
\end{tabular}

Table 2. Conditions for fall and spring simulation experiments.

\begin{tabular}{lcccc}
\hline $\begin{array}{l}\text { Duration } \\
\text { (days) }\end{array}$ & $\begin{array}{c}\text { Light } \\
\text { temp } \\
\left({ }^{\circ} \mathrm{C}\right)\end{array}$ & $\begin{array}{c}\text { Dark } \\
\text { temp } \\
\left({ }^{\circ} \mathrm{C}\right)\end{array}$ & $\begin{array}{c}P P F \\
\left(\mu \mathrm{mol} \cdot \mathrm{m}^{-2} \cdot \mathrm{s}^{-1}\right)\end{array}$ & $\begin{array}{c}\text { Photoperiod } \\
(\mathrm{h})\end{array}$ \\
\hline $\begin{array}{l}\text { Fall simulation } \\
2\end{array}$ & & & & \\
4 & 30.0 & --- & 400 & 24 \\
3 & 26.5 & 15.5 & 350 & 21 \\
3 & 23.0 & 13.0 & 300 & 18 \\
5 & 19.5 & 10.5 & 250 & 15 \\
Spring simulation & 16.0 & 8.0 & 200 & 12 \\
3 & & & & \\
3 & 16.0 & 8.0 & 200 & 12 \\
4 & 19.5 & 10.5 & 250 & 15 \\
3 & 23.0 & 13.0 & 300 & 18 \\
2 & 26.5 & 15.5 & 350 & 21 \\
\hline & 30.0 & --- & 400 & 24 \\
\hline
\end{tabular}

(2-phenylethyl GS; GNS) in addition to the same GSs found in leaves. Roots contained the same GSs as stems and also had the aliphatic GS glucoerucin (4-methylthiobutyl GS; GE), although GI was not detected at $12^{\circ} \mathrm{C}$.

Aliphatic GSs comprised $90 \%\left(22^{\circ} \mathrm{C}\right)$ to $95 \%\left(32^{\circ} \mathrm{C}\right)$ of total
GSs. GNP, PRO, and SN were the predominant GSs in all temperature treatments, each comprising $17 \%$ to $43 \%$ of total GSs. Concentrations of total and aliphatic GSs in leaves were dramatically elevated in the high and low temperature treatments. Total GSs were $44 \%$ and $114 \%$ higher at $12{ }^{\circ} \mathrm{C}$ and $32^{\circ} \mathrm{C}$ respectively compared to those at $22^{\circ} \mathrm{C}$. Aliphatic GSs increased by $45 \%$ and $125 \%$ at 12 and $32{ }^{\circ} \mathrm{C}$ respectively compared to those at $22^{\circ} \mathrm{C}$. GR ranged from $6 \%$ to $14 \%$ of total GSs and was over 5-fold higher in leaves of plants grown at 32 than at $22^{\circ} \mathrm{C}$.

Indole GSs in leaves did not vary with temperature. In stems, however, indole GSs increased linearly with temperature. At 32 ${ }^{\circ} \mathrm{C}$, indole GS concentrations in stems were $138 \%$ higher than in stems grown at $12^{\circ} \mathrm{C}$. GNP, PRO, and $\mathrm{SN}$ were the predominant GSs in stems in all temperature treatments. As with leaves, GNP concentrations in stems increased with temperature, and were $105 \%$ higher in stems from plants grown at $32{ }^{\circ} \mathrm{C}$ than from plants grown at $22^{\circ} \mathrm{C}$.

Total GS concentrations in roots were higher than in leaves or stems. Total GSs varied quadratically with temperature and were highest in roots of plants grown at $22{ }^{\circ} \mathrm{C}$. Indole GSs made up a larger portion of total GSs in roots than in leaves or stems. At 22 ${ }^{\circ} \mathrm{C}, 41 \%$ of total GSs were indole GSs. Indole GSs increased with temperature whereas aliphatic GSs decreased. GNS accounted for $28 \%$ of total GSs at $22^{\circ} \mathrm{C}$. GE was the predominant aliphatic GS whereas $4 \mathrm{MGB}$ and NGB were the predominant indole GSs. NGB concentrations increased with temperature but $4 \mathrm{MGB}$ concentrations decreased.

GLUCOSINOLATES FROM PLANTS GROWN AT DIFFERENT LEVELS OF PPF . The same spectrum of GSs were detected in this experiment as in the temperature experiment. Major GSs in leaves at all $P P F$ levels were GNP, PRO, and SN. Total GS concentrations in leaves decreased with increasing $P P F$ (Table 4). Most of this decrease was due to the decrease in aliphatic GSs from $7.8 \pm$ $0.3 \mu \mathrm{mol} \cdot \mathrm{g}^{-1}$ dry weight (mean $\pm \mathrm{SE}$ ) at $200 \mu \mathrm{mol} \cdot \mathrm{m}^{-2} \cdot \mathrm{s}^{-1}$ to 6.2 $\pm 0.3 \mu \mathrm{mol} \cdot \mathrm{g}^{-1}$ at $400 \mu \mathrm{mol} \cdot \mathrm{m}^{-2} \cdot \mathrm{s}^{-1}$, a $21 \%$ reduction. Total GSs decreased $20 \%$ from 200 to $400 \mu \mathrm{mol} \cdot \mathrm{m}^{-2} \cdot \mathrm{s}^{-1}$. GR concentrations were highest at $300 \mu \mathrm{mol} \cdot \mathrm{m}^{-2} \cdot \mathrm{s}^{-1}(6 \%$ of total GSs) and were $50 \%$ lower at both 200 and $400 \mu \mathrm{mol} \cdot \mathrm{m}^{-2 \cdot \mathrm{s}^{-1}}$.

Table 3. Mean glucosinolate (GS) concentrations in rapid-cycling Brassica oleracea grown at different temperatures.

\begin{tabular}{|c|c|c|c|c|c|c|c|c|c|c|c|c|c|c|}
\hline \multirow{3}{*}{$\begin{array}{l}\text { Temp } \\
\left({ }^{\circ} \mathrm{C}\right)\end{array}$} & \multicolumn{14}{|c|}{ Glucosinolate $\left(\mu \mathrm{mol} \cdot \mathrm{g}^{-1}\right.$ dry wt $\left.\pm \mathrm{SE}\right)$} \\
\hline & Total & Aliphatic & Indole & & & & & & & & & & & \\
\hline & GSs & GSs & GSs & $\mathrm{GI}^{2}$ & GNP & GR & PRO & GE & $\mathrm{SN}$ & 4OHGB & GB & 4MGB & NGB & GNS \\
\hline \multicolumn{15}{|l|}{ Leaves } \\
\hline 12 & $10.2 \pm 0.2$ & $9.3 \pm 0.2$ & $0.8 \pm 0.1$ & $0.5 \pm 0.0$ & $3.6 \pm 0.4$ & $0.8 \pm 0.1$ & $1.9 \pm 0.2$ & NDy & $2.5 \pm 0.1$ & $0.1 \pm 0.0$ & $0.4 \pm 0.0$ & $0.3 \pm 0.0$ & $0.0 \pm 0.0$ & ND \\
\hline 22 & $7.1 \pm 0.4$ & $6.4 \pm 0.3$ & $0.7 \pm 0.1$ & $0.3 \pm 0.0$ & $2.2 \pm 0.1$ & $0.4 \pm 0.0$ & $1.5 \pm 0.2$ & ND & $2.1 \pm 0.1$ & $0.1 \pm 0.0$ & $0.3 \pm 0.0$ & $0.3 \pm 0.0$ & $0.1 \pm 0.0$ & ND \\
\hline 32 & $15.2 \pm 1.5$ & $14.4 \pm 1.4$ & $0.9 \pm 0.1$ & $0.7 \pm 0.1$ & $6.5 \pm 0.8$ & $2.1 \pm 0.2$ & $2.6 \pm 0.4$ & ND & $2.8 \pm 0.3$ & $0.3 \pm 0.0$ & $0.3 \pm 0.0$ & $0.1 \pm 0.0$ & $0.2 \pm 0.0$ & ND \\
\hline Linear & ** & $* *$ & NS & NS & ** & $* *$ & $*$ & NS & NS & ** & * & NS & ** & --- \\
\hline Quadratic & $* *$ & $* *$ & NS & $* *$ & $* *$ & $* *$ & $*$ & NS & $*$ & $* *$ & $* *$ & $* *$ & NS & --- \\
\hline \multicolumn{15}{|l|}{ Stems } \\
\hline 12 & $14.3 \pm 0.6$ & $13.1 \pm 0.6$ & $0.8 \pm 0.0$ & $1.3 \pm 0.1$ & $4.4 \pm 0.5$ & $1.5 \pm 0.2$ & $2.7 \pm 0.3$ & ND & $3.2 \pm 0.1$ & $0.1 \pm 0.0$ & $0.5 \pm 0.0$ & $0.1 \pm 0.0$ & $0.1 \pm 0.0$ & $0.5 \pm 0.0$ \\
\hline 22 & $13.4 \pm 0.5$ & $11.7 \pm 0.4$ & $1.1 \pm 0.1$ & $0.8 \pm 0.1$ & $4.0 \pm 0.4$ & $0.9 \pm 0.0$ & $2.7 \pm 0.2$ & ND & $3.2 \pm 0.2$ & $0.2 \pm 0.0$ & $0.4 \pm 0.0$ & $0.2 \pm 0.0$ & $0.3 \pm 0.0$ & $0.6 \pm 0.0$ \\
\hline 32 & $16.7 \pm 2.2$ & $14.3 \pm 1.9$ & $1.9 \pm 0.2$ & $0.4 \pm 0.1$ & $8.2 \pm 1.5$ & $1.4 \pm 0.2$ & $1.9 \pm 0.2$ & ND & $2.4 \pm 0.4$ & $0.5 \pm 0.1$ & $0.3 \pm 0.0$ & $0.3 \pm 0.0$ & $1.0 \pm 0.1$ & $0.4 \pm 0.1$ \\
\hline Linear & NS & NS & $* *$ & $* *$ & ** & NS & $*$ & --- & $*$ & ** & ** & $* *$ & $* *$ & NS \\
\hline Quadratic & NS & NS & NS & NS & NS & $*$ & NS & --- & NS & NS & NS & NS & $*$ & $*$ \\
\hline \multicolumn{15}{|l|}{ Roots } \\
\hline 12 & $21.5 \pm 1.3$ & $9.2 \pm 0.6$ & $6.8 \pm 0.4$ & $\mathrm{ND}$ & $1.0 \pm 0.1$ & $0.2 \pm 0.0$ & $2.0 \pm 0.2$ & $5.1 \pm 0.4$ & $1.0 \pm 0.1$ & $0.4 \pm 0.1$ & $1.1 \pm 0.1$ & $3.6 \pm 0.3$ & $1.7 \pm 0.1$ & $5.4 \pm 0.5$ \\
\hline 22 & $31.4 \pm 1.3$ & $9.9 \pm 0.5$ & $12.8 \pm 0.8$ & $0.1 \pm 0.0$ & $1.6 \pm 0.1$ & $0.2 \pm 0.0$ & $2.3 \pm 0.1$ & $4.5 \pm 0.4$ & $1.2 \pm 0.1$ & $2.4 \pm 0.2$ & $1.8 \pm 0.2$ & $3.1 \pm 0.2$ & $5.6 \pm 0.7$ & $8.8 \pm 0.3$ \\
\hline 32 & $21.7 \pm 1.4$ & $6.2 \pm 0.6$ & $13.0 \pm 1.1$ & $0.0 \pm 0.0$ & $1.9 \pm 0.3$ & $0.3 \pm 0.1$ & $1.1 \pm 0.2$ & $2.4 \pm 0.3$ & $0.5 \pm 0.1$ & $1.9 \pm 0.4$ & $0.8 \pm 0.1$ & $1.7 \pm 0.2$ & $8.7 \pm 0.8$ & $2.4 \pm 0.3$ \\
\hline Linear & NS & $* *$ & $* *$ & NS & ** & NS & ** & $* *$ & ** & $* *$ & $*$ & ** & $* *$ & $* *$ \\
\hline Quadratic & $* *$ & $* *$ & $* *$ & $* *$ & NS & NS & $* *$ & NS & $* *$ & $* *$ & $* *$ & NS & NS & $* *$ \\
\hline
\end{tabular}

${ }^{2} \mathrm{GI}=$ glucoiberin, $\mathrm{GNP}=$ gluconapin, $\mathrm{GR}=$ glucoraphanin, $\mathrm{PRO}=$ progoitrin, $\mathrm{GE}=$ glucoerucin; $\mathrm{SN}=$ sinigrin, 4OHGB = 4-hydroxyglucobrassicin, $\mathrm{GB}=$ glucobrassicin, 4MGB = 4methoxyglucobrassicin, $\mathrm{NGB}=$ neoglucobrassicin, $\mathrm{GNS}=$ gluconasturtiin .

${ }^{\mathrm{ND}}=$ not detected.

Ns,",***Nonsignificant or significant at $P \leq 0.05$ or 0.01 , respectively. 
In stems, total GSs, aliphatic GSs as a group, GI, GR, PRO, and $\mathrm{SN}$ had negative quadratic relationships with $P P F$. In contrast, the group of indole GSs and the individual GSs GB, 4MGB, NGB, and GNS had positive quadratic relationships with $P P F$. There were no differences in total or indole GSs in roots but aliphatic GSs, GNP, GR, PRO, GE, SN, and 4OHGB had negative quadratic relationships with $P P F$. Gluconasturtiin in roots increased linearly with $P P F$.

GLUCOSINOLATES FROM PLANTS GROWN WITH DIFFERENT PHOTOPERIODS. GI was not detected in leaves or roots from plants grown under a $12 \mathrm{~h}$ photoperiod nor in roots of plants grown under 18 h light (Table 5). Otherwise the GS profiles for leaves, stems, and roots were the same as those determined in the temperature and $P P F$ experiments. GI, GR, and $4 \mathrm{MGB}$ in leaves increased with photoperiod, but concentrations of these GSs were too low to affect total, aliphatic, or indole GSs.

Total glucosinolates in stems had a positive linear and negative quadratic relationship with photoperiod, primarily due to the same response in aliphatic GSs. The SN concentration in stems from the $18 \mathrm{~h}$ photoperiod was $45 \%$ and $25 \%$ higher than that in stems grown with 12 and $24 \mathrm{~h}$ light, respectively. The group of indole GSs was not affected by photoperiod, although the individual indole GSs 4OHGB and NGB were quadratically related with photoperiod. Total GSs, aliphatic GSs, GR PRO, GE, NGB, and GNS in roots increased linearly with photoperiod. GE, NGB, and GNS were present at the highest concentrations. GE comprised

Table 4. Mean glucosinolate (GS) concentrations in rapid-cycling Brassica oleracea grown at different levels of photosynthetic photon flux ( $P P F$ ).

\begin{tabular}{|c|c|c|c|c|c|c|c|c|c|c|c|c|c|c|}
\hline \multirow[b]{2}{*}{$\begin{array}{l}P P F \\
\left(\mu \mathrm{mol} \cdot \mathrm{m}^{-2} \cdot \mathrm{s}^{-1}\right)\end{array}$} & \multicolumn{14}{|c|}{ Glucosinolate $\left(\mu \mathrm{mol} \cdot \mathrm{g}^{-1}\right.$ dry $\left.\mathrm{wt} \pm \mathrm{SE}\right)$} \\
\hline & $\begin{array}{l}\text { Total } \\
\text { GSs }\end{array}$ & $\begin{array}{c}\text { Aliphatic } \\
\text { GSs }\end{array}$ & $\begin{array}{l}\text { Indole } \\
\text { GSs }\end{array}$ & $\mathrm{GI}^{\mathrm{z}}$ & GNP & GR & PRO & GE & SN & 4OHGB & GB & 4MGB & NGB & GNS \\
\hline \multicolumn{15}{|l|}{ Leaves } \\
\hline 200 & $8.4 \pm 0.2$ & $7.8 \pm 0.3$ & $0.6 \pm 0.1$ & $0.3 \pm 0.0$ & $2.6 \pm 0.2$ & $0.2 \pm 0.0$ & $2.0 \pm 0.3$ & NDy & $2.7 \pm 0.1$ & $0.1 \pm 0.0$ & $0.3 \pm 0.0$ & $0.2 \pm 0.0$ & $0.1 \pm 0.0$ & ND \\
\hline 300 & $7.1 \pm 0.4$ & $6.4 \pm 0.3$ & $0.7 \pm 0.1$ & $0.3 \pm 0.0$ & $2.2 \pm 0.1$ & $0.4 \pm 0.0$ & $1.5 \pm 0.2$ & ND & $2.1 \pm 0.2$ & $0.1 \pm 0.0$ & $0.3 \pm 0.0$ & $0.3 \pm 0.0$ & $0.1 \pm 0.0$ & ND \\
\hline 400 & $6.7 \pm 0.3$ & $6.2 \pm 0.3$ & $0.4 \pm 0.0$ & $0.3 \pm 0.0$ & $2.2 \pm 0.3$ & $0.2 \pm 0.0$ & $1.4 \pm 0.2$ & ND & $2.2 \pm 0.1$ & $0.1 \pm 0.0$ & $0.2 \pm 0.0$ & $0.1 \pm 0.0$ & $0.1 \pm 0.0$ & ND \\
\hline Linear & ** & ** & $*$ & NS & NS & NS & NS & --- & ** & NS & NS & $*$ & NS & --- \\
\hline Quadratic & NS & NS & $*$ & NS & NS & $* *$ & NS & --- & $*$ & NS & NS & $*$ & NS & --- \\
\hline \multicolumn{15}{|l|}{ Stems } \\
\hline 200 & $12.9 \pm 0.9$ & $9.2 \pm 0.7$ & $1.7 \pm 0.1$ & $0.4 \pm 0.1$ & $3.6 \pm 0.4$ & $0.3 \pm 0.0$ & $2.1 \pm 0.2$ & ND & $2.7 \pm 0.2$ & $0.3 \pm 0.0$ & $0.5 \pm 0.1$ & $0.4 \pm 0.0$ & $1.0 \pm 0.1$ & $2.0 \pm 0.1$ \\
\hline 300 & $13.4 \pm 0.5$ & $11.7 \pm 0.4$ & $1.1 \pm 0.1$ & $0.8 \pm 0.1$ & $4.0 \pm 0.4$ & $0.9 \pm 0.0$ & $2.7 \pm 0.2$ & ND & $3.2 \pm 0.2$ & $0.2 \pm 0.0$ & $0.4 \pm 0.0$ & $0.2 \pm 0.0$ & $0.3 \pm 0.0$ & $0.6 \pm 0.0$ \\
\hline 400 & $10.7 \pm 0.3$ & $8.1 \pm 0.3$ & $1.5 \pm 0.1$ & $0.3 \pm 0.0$ & $3.3 \pm 0.3$ & $0.3 \pm 0.0$ & $1.7 \pm 0.2$ & ND & $2.4 \pm 0.1$ & $0.2 \pm 0.0$ & $0.5 \pm 0.0$ & $0.3 \pm 0.0$ & $0.5 \pm 0.0$ & $1.2 \pm 0.1$ \\
\hline Linear & * & NS & NS & NS & NS & NS & NS & --- & NS & NS & NS & NS & NS & NS \\
\hline Quadratic & * & $* *$ & $* *$ & $* *$ & NS & $* *$ & $*$ & --- & $* *$ & NS & $*$ & $* *$ & $* *$ & $* *$ \\
\hline \multicolumn{15}{|l|}{ Roots } \\
\hline 200 & $29.0 \pm 2.6$ & $6.7 \pm 0.5$ & $13.8 \pm 1.8$ & $0.1 \pm 0.0$ & $1.1 \pm 0.1$ & $0.2 \pm 0.0$ & $1.7 \pm 0.2$ & $2.8 \pm 0.2$ & $0.8 \pm 0.1$ & $1.7 \pm 0.3$ & $1.6 \pm 0.3$ & $4.1 \pm 0.4$ & $6.5 \pm 0.9$ & $8.4 \pm 0.5$ \\
\hline 300 & $31.4 \pm 1.3$ & $9.9 \pm 0.5$ & $12.8 \pm 0.8$ & $0.1 \pm 0.0$ & $1.6 \pm 0.1$ & $0.2 \pm 0.0$ & $2.3 \pm 0.1$ & $4.5 \pm 0.4$ & $1.2 \pm 0.1$ & $2.4 \pm 0.2$ & $1.8 \pm 0.2$ & $3.1 \pm 0.2$ & $5.6 \pm 0.4$ & $8.8 \pm 0.3$ \\
\hline 400 & $33.1 \pm 1.6$ & $7.7 \pm 0.5$ & $15.2 \pm 0.9$ & $0.1 \pm 0.0$ & $1.1 \pm 0.1$ & $0.2 \pm 0.0$ & $1.6 \pm 0.1$ & $3.7 \pm 0.4$ & $1.0 \pm 0.1$ & $1.7 \pm 0.2$ & $19 \pm 0.2$ & $3.6 \pm 0.3$ & $7.9 \pm 0.6$ & $10.2 \pm 0.4$ \\
\hline Linear & NS & NS & NS & NS & NS & NS & NS & NS & NS & NS & NS & NS & NS & * \\
\hline Quadratic & NS & $* *$ & NS & NS & $* *$ & $* *$ & $* *$ & $* *$ & $*$ & $*$ & NS & NS & NS & NS \\
\hline
\end{tabular}

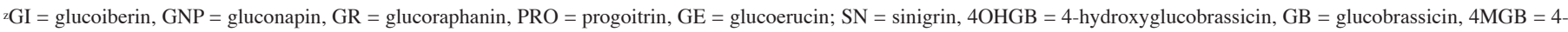
methoxyglucobrassicin, NGB = neoglucobrassicin, GNS = gluconasturtiin.

yND $=$ not detected.

Ns,",***Nonsignificant or significant at $P \leq 0.05$ or 0.01 , respectively.

Table 5. Mean glucosinolate (GS) concentrations in rapid-cycling Brassica oleracea grown with different photoperiods.

\begin{tabular}{|c|c|c|c|c|c|c|c|c|c|c|c|c|c|c|}
\hline \multirow[b]{2}{*}{$\begin{array}{l}\text { Photoperiod } \\
\text { (h) }\end{array}$} & \multicolumn{14}{|c|}{ Glucosinolate $\left(\mu \mathrm{mol} \cdot \mathrm{g}^{-1}\right.$ dry $\left.\mathrm{wt} \pm \mathrm{SE}\right)$} \\
\hline & $\begin{array}{l}\text { Total } \\
\text { GSs }\end{array}$ & $\begin{array}{l}\text { Aliphatic } \\
\text { GSs }\end{array}$ & $\begin{array}{l}\text { Indole } \\
\text { GSs }\end{array}$ & $\mathrm{GI}^{2}$ & GNP & GR & PRO & GE & SN & 4OHGB & GB & 4MGB & NGB & GNS \\
\hline \multicolumn{15}{|l|}{ Leaves } \\
\hline 12 & $6.3 \pm 0.6$ & $5.7 \pm 0.5$ & $0.6 \pm 0.1$ & ND & $2.4 \pm 0.4$ & $0.1 \pm 0.0$ & $1.3 \pm 0.2$ & NDy & $1.9 \pm 0.1$ & $0.1 \pm 0.0$ & $0.3 \pm 0.1$ & $0.2 \pm 0.0$ & $0.1 \pm 0.0$ & ND \\
\hline 18 & $6.7 \pm 0.3$ & $6.1 \pm 0.3$ & $0.6 \pm 0.0$ & $0.1 \pm 0.0$ & $2.5 \pm 0.2$ & $0.1 \pm 0.0$ & $1.3 \pm 0.1$ & ND & $2.2 \pm 0.1$ & $0.1 \pm 0.0$ & $0.3 \pm 0.0$ & $0.2 \pm 0.0$ & $0.1 \pm 0.0$ & ND \\
\hline 24 & $7.1 \pm 0.4$ & $6.4 \pm 0.3$ & $0.7 \pm 0.1$ & $0.3 \pm 0.0$ & $2.2 \pm 0.1$ & $0.4 \pm 0.0$ & $1.5 \pm 0.2$ & ND & $2.1 \pm 0.2$ & $0.1 \pm 0.0$ & $0.3 \pm 0.0$ & $0.3 \pm 0.0$ & $0.1 \pm 0.0$ & ND \\
\hline Linear & NS & NS & NS & $* *$ & NS & $* *$ & NS & --- & NS & NS & NS & $*$ & NS & --- \\
\hline Quadratic & NS & NS & NS & NS & NS & $* *$ & NS & -- & NS & NS & NS & NS & NS & --- \\
\hline \multicolumn{15}{|l|}{ Stems } \\
\hline 12 & $9.4 \pm 1.0$ & $7.6 \pm 0.9$ & $1.2 \pm 0.1$ & $0.0 \pm 0.0$ & $3.0 \pm 0.5$ & $0.1 \pm 0.0$ & $2.2 \pm 0.4$ & ND & $2.2 \pm 0.3$ & $0.2 \pm 0.0$ & $0.4 \pm 0.1$ & $0.2 \pm 0.0$ & $0.4 \pm 0.1$ & $0.6 \pm 0.1$ \\
\hline 18 & $14.1 \pm 0.4$ & $12.7 \pm 0.3$ & $1.0 \pm 0.0$ & $0.4 \pm 0.1$ & $4.8 \pm 0.2$ & $0.4 \pm 0.0$ & $3.0 \pm 0.3$ & ND & $4.0 \pm 0.1$ & $0.1 \pm 0.0$ & $0.4 \pm 0.0$ & $0.2 \pm 0.0$ & $0.3 \pm 0.0$ & $0.4 \pm 0.0$ \\
\hline 24 & $13.4 \pm 0.5$ & $11.7 \pm 0.4$ & $1.1 \pm 0.1$ & $0.9 \pm 0.1$ & $4.0 \pm 0.4$ & $0.9 \pm 0.0$ & $2.7 \pm 0.2$ & ND & $3.2 \pm 0.2$ & $0.2 \pm 0.0$ & $0.4 \pm 0.0$ & $0.2 \pm 0.0$ & $0.3 \pm 0.0$ & $0.6 \pm 0.0$ \\
\hline Linear & $* *$ & ** & NS & ** & NS & ** & NS & --- & ** & NS & NS & NS & NS & NS \\
\hline Quadratic & $* *$ & $* *$ & NS & NS & $*$ & $*$ & NS & -- & $* *$ & $*$ & NS & NS & $*$ & $* *$ \\
\hline \multicolumn{15}{|l|}{ Roots } \\
\hline 12 & $22.6 \pm 1.8$ & $5.3 \pm 0.5$ & $11.4 \pm 1.0$ & ND & $0.9 \pm 0.2$ & $0.1 \pm 0.0$ & $1.8 \pm 0.1$ & $2.0 \pm 0.3$ & $0.6 \pm 0.1$ & $2.6 \pm 0.3$ & $1.4 \pm 0.2$ & $3.5 \pm 0.5$ & $3.9 \pm 0.2$ & $6.0 \pm 0.4$ \\
\hline 18 & $26.0 \pm 1.4$ & $8.5 \pm 0.5$ & $11.1 \pm 0.7$ & ND & $2.0 \pm 0.2$ & $0.2 \pm 0.0$ & $1.8 \pm 0.1$ & $3.3 \pm 0.3$ & $1.3 \pm 0.1$ & $1.7 \pm 0.1$ & $1.4 \pm 0.1$ & $3.2 \pm 0.2$ & $4.8 \pm 0.4$ & $6.4 \pm 0.3$ \\
\hline 24 & $31.4 \pm 1.3$ & $9.9 \pm 0.5$ & $12.8 \pm 0.8$ & $0.1 \pm 0.0$ & $1.6 \pm 0.1$ & $0.2 \pm 0.0$ & $2.3 \pm 0.1$ & $4.5 \pm 0.4$ & $1.2 \pm 0.1$ & $2.4 \pm 0.2$ & $1.8 \pm 0.2$ & $3.1 \pm 0.2$ & $5.6 \pm 0.4$ & $8.8 \pm 0.3$ \\
\hline Linear & ** & ** & NS & NS & ** & ** & $*$ & $* *$ & ** & NS & NS & NS & $* *$ & $* *$ \\
\hline Quadratic & NS & NS & NS & NS & $* *$ & NS & NS & NS & $* *$ & $*$ & NS & NS & NS & NS \\
\hline
\end{tabular}

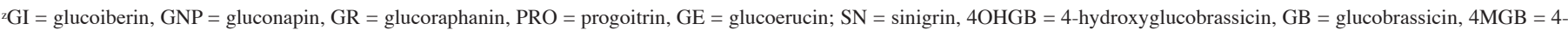
methoxyglucobrassicin, NGB = neoglucobrassicin, GNS = gluconasturtiin. yND $=$ not detected.

ss, ${ }^{*, *}$ Nonsignificant or significant at $P \leq 0.05$ or 0.01 , respectively. 
Table 6. Mean glucosinolate (GS) concentrations in rapid-cycling Brassica oleracea grown in simulated fall or spring conditions.

\begin{tabular}{|c|c|c|c|c|c|c|c|c|c|c|c|c|c|c|}
\hline \multirow[b]{2}{*}{ Season } & \multicolumn{14}{|c|}{ Glucosinolate $\left(\mu \mathrm{mol} \cdot \mathrm{g}^{-1}\right.$ dry wt $\left.\pm \mathrm{SE}\right)$} \\
\hline & $\begin{array}{l}\text { Total } \\
\text { GSs }\end{array}$ & $\begin{array}{c}\text { Aliphatic } \\
\text { GSs }\end{array}$ & $\begin{array}{l}\text { Indole } \\
\text { GSs }\end{array}$ & $\mathrm{GI}^{\mathrm{z}}$ & GNP & GR & PRO & GE & SN & 4OHGB & GB & 4MGB & NGB & GNS \\
\hline \multicolumn{15}{|l|}{ Leaves } \\
\hline Fall & $6.8 \pm 0.4$ & $6.1 \pm 0.4$ & $0.7 \pm 0.1$ & $0.1 \pm 0.0$ & $2.5 \pm 0.4$ & $0.1 \pm 0.0$ & $1.4 \pm 0.2$ & NDy & $2.1 \pm 0.2$ & $0.1 \pm 0.0$ & $0.3 \pm 0.0$ & $0.2 \pm 0.0$ & $0.1 \pm 0.0$ & ND \\
\hline \multirow[t]{2}{*}{ Spring } & $9.0 \pm 0.3$ & $8.4 \pm 0.3$ & $0.5 \pm 0.0$ & $0.2 \pm 0.0$ & $3.4 \pm 0.4$ & $0.4 \pm 0.1$ & $1.7 \pm 0.2$ & ND & $2.7 \pm 0.1$ & $0.1 \pm 0.0$ & $0.3 \pm 0.0$ & $0.1 \pm 0.0$ & $0.1 \pm 0.0$ & ND \\
\hline & $*$ & $*$ & NS & $*$ & NS & $*$ & NS & --- & * & NS & NS & $*$ & $*$ & --- \\
\hline \multicolumn{15}{|l|}{ Stems } \\
\hline Fall & $11.3 \pm 0.8$ & $8.8 \pm 0.5$ & $1.6 \pm 0.1$ & $0.2 \pm 0.0$ & $3.6 \pm 0.6$ & $0.3 \pm 0.0$ & $2.0 \pm 0.4$ & ND & $2.6 \pm 0.1$ & $0.3 \pm 0.0$ & $0.7 \pm 0.0$ & $0.2 \pm 0.0$ & $0.4 \pm 0.1$ & $0.9 \pm 0.1$ \\
\hline \multirow[t]{2}{*}{ Spring } & $19.0 \pm 1.0$ & $16.3 \pm 0.9$ & $1.7 \pm 0.1$ & $0.6 \pm 0.0$ & $7.8 \pm 1.0$ & $0.8 \pm 0.1$ & $3.0 \pm 0.5$ & ND & $4.1 \pm 0.2$ & $0.6 \pm 0.0$ & $0.4 \pm 0.1$ & $0.3 \pm 0.0$ & $0.5 \pm 0.0$ & $0.9 \pm 0.1$ \\
\hline & $*$ & $*$ & NS & * & $*$ & * & NS & --- & * & $*$ & $*$ & NS & NS & NS \\
\hline \multicolumn{15}{|l|}{ Roots } \\
\hline Fall & $27.7 \pm 2.8$ & $7.1 \pm 0.6$ & $12.6 \pm 1.5$ & $0.0 \pm 0.0$ & $1.4 \pm 0.2$ & $0.2 \pm 0.0$ & $1.9 \pm 0.2$ & $2.9 \pm 0.4$ & $0.8 \pm 0.0$ & $2.2 \pm 0.4$ & $2.1 \pm 0.3$ & $4.1 \pm 0.6$ & $4.2 \pm 0.4$ & $7.9 \pm 0.9$ \\
\hline \multirow[t]{2}{*}{ Spring } & $29.0 \pm 1.0$ & $8.8 \pm 0.5$ & $13.6 \pm 0.3$ & $0.1 \pm 0.0$ & $2.2 \pm 0.3$ & $0.2 \pm 0.0$ & $2.3 \pm 0.2$ & $2.8 \pm 0.2$ & $1.2 \pm 0.2$ & $2.3 \pm 0.2$ & $1.7 \pm 0.1$ & $2.9 \pm 0.4$ & $6.7 \pm 0.2$ & $6.6 \pm 0.5$ \\
\hline & NS & $*$ & NS & * & $*$ & NS & NS & NS & $*$ & NS & NS & $*$ & * & NS \\
\hline
\end{tabular}

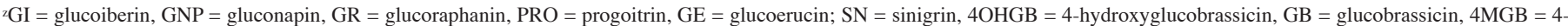
methoxyglucobrassicin, NGB = neoglucobrassicin, GNS = gluconasturtiin.

y $\mathrm{ND}=$ not detected.

ws, Nonsignificant or significant at $P \leq 0.05$, respectively.

9\% (12-h photoperiod) to $14 \%$ (24-h photoperiod) of total GSs, NGB comprised $17 \%$ (12- and 24-h photoperiods) to $18 \%$ (18 h photoperiod), and GNS varied from 25\% (18-h photoperiod) to $28 \%$ (24-h photoperiod) of total GSs.

GLUCOSINOLATES IN THE FALL SIMULATION AND SPRING SIMULATION EXPERIMENTS. The spectrum of GSs detected in leaves, stems, and roots was identical to that determined in the temperature and $P P F$ experiments (Table 6). Total and aliphatic GSs, GI, GR, SN, and NGB in leaves from the spring simulation were higher than in leaves from the fall simulation. Total GSs and aliphatic GSs from leaves grown in the spring simulation were $32 \%$ and $38 \%$ higher, respectively, than in the fall simulation. Concentrations of $4 \mathrm{MGB}$ were $44 \%$ lower in leaves from the spring simulation than in the fall simulation.

GSs in stems followed a similar pattern although the differences were more pronounced. Total GSs in stems of the spring simulation were $68 \%$ higher than in those from the fall simulation. Aliphatic GSs in stems from the spring simulation were

Table 7. Mean myrosinase activity in crude extracts from rapid-cycling Brassica oleracea grown at different temperatures ${ }^{\mathrm{z}}$.

\begin{tabular}{lcc}
\hline \multirow{2}{*}{$\begin{array}{l}\text { Temp } \\
\left({ }^{\circ} \mathrm{C}\right)\end{array}$} & \multicolumn{2}{c}{ Activity } \\
\cline { 2 - 3 } Leaves & Activity-FW & $\begin{array}{c}\text { Specific activity } \\
(\mathrm{U} / \mathrm{mg} \pm \mathrm{SE})\end{array}$ \\
12 & $43.5 \pm 4.4$ & $12.1 \pm 2.2$ \\
22 & $29.6 \pm 2.0$ & $18.5 \pm 3.5$ \\
32 & $38.1 \pm 1.6$ & $16.5 \pm 2.0$ \\
Linear & $\mathrm{NS}$ & $\mathrm{NS}$ \\
Quadratic & $* *$ & $\mathrm{NS}$ \\
Stems & & $23.6 \pm 1.3$ \\
12 & $45.2 \pm 4.8$ & $20.9 \pm 2.6$ \\
22 & $24.3 \pm 2.8$ & $23.5 \pm 0.8$ \\
32 & $39.7 \pm 5.1$ & $\mathrm{NS}$ \\
Linear & $\mathrm{NS}$ & $\mathrm{NS}$ \\
Quadratic & $* *$ & $33.6 \pm 3.0$ \\
Roots & & $28.3 \pm 6.1$ \\
12 & $37.4 \pm 3.8$ & $28.9 \pm 4.2$ \\
22 & $45.3 \pm 5.7$ & $\mathrm{NS}$ \\
32 & $39.3 \pm 6.6$ & $\mathrm{NS}$ \\
Linear & $\mathrm{NS}$ & $\mathrm{NS}$ \\
Quadratic & $\mathrm{NS}$ & \\
\hline
\end{tabular}

ss,, ,**Nonsignificant or significant at $P \leq 0.05$ or 0.01 , respectively.
$85 \%$ higher than in those from the fall simulation. GI, GNP, GR, $\mathrm{SN}$, and $4 \mathrm{OHGB}$ were all higher in the spring simulation than in the fall simulation. GB was $43 \%$ lower in stems from the spring simulation compared to the fall simulation.

Several GSs in roots also were higher in the spring simulation than in the fall simulation. Aliphatic GSs, GI, GNP, SN, and NGB were higher in the spring simulation than in the fall simulation. Aliphatic GSs were 24\% higher. Concentrations of 4MGB were $29 \%$ lower in the spring simulation compared to the fall simulation. Thus in leaves and roots, 4MGB was highest in the fall simulation and NGB was highest in the spring simulation.

ACTIVITY OF MYROSINASE FROM PLANTS GROWN AT DIFFERENT TEMPERATURES. When calculated on a fresh weight basis (activityFW), there was a positive quadratic relationship of myrosinase activity in crude extracts from leaves and stems with temperature (Table 7). Activity-FW in leaves produced at 12 and $32{ }^{\circ} \mathrm{C}$ was $47 \%$ and $29 \%$ higher respectively compared to activity-FW in

Table 8. Mean myrosinase activity in crude extracts from rapid-cycling Brassica oleracea grown under different levels of photosynthetic photon flux $(P P F)$.

\begin{tabular}{lcc}
\hline & \multicolumn{2}{c}{ Activity } \\
\cline { 2 - 3 }$(\mu P F$ & $\begin{array}{c}\text { Activity-FW } \\
(\mathrm{U} / \mathrm{g} \pm \mathrm{SE})\end{array}$ & $\begin{array}{c}\text { Specific activity } \\
(\mathrm{U} / \mathrm{mg} \pm \mathrm{SE})\end{array}$ \\
\hline Leaves & & \\
200 & $42.4 \pm 3.8$ & $34.2 \pm 6.1$ \\
300 & $29.6 \pm 2.0$ & $18.5 \pm 3.5$ \\
400 & $38.0 \pm 5.2$ & $19.6 \pm 4.4$ \\
Linear & $\mathrm{NS}$ & $*$ \\
Quadratic & $* *$ & $\mathrm{NS}$ \\
Stems & & $20.8 \pm 5.3$ \\
200 & $24.3 \pm 1.8$ & $18.3 \pm 3.3$ \\
300 & $24.3 \pm 2.8$ & $23.1 \pm 2.3$ \\
400 & $25.5 \pm 1.9$ & $\mathrm{NS}$ \\
Linear & $\mathrm{NS}$ & $\mathrm{NS}$ \\
Quadratic & $\mathrm{NS}$ & $31.3 \pm 5.4$ \\
Roots & & $28.3 \pm 6.1$ \\
200 & $30.9 \pm 3.6$ & $28.4 \pm 3.2$ \\
300 & $45.3 \pm 5.7$ & $\mathrm{NS}$ \\
400 & $32.5 \pm 5.4$ & $\mathrm{NS}$ \\
Linear & $\mathrm{NS}$ & \\
Quadratic & $* *$ &
\end{tabular}

ss,*,**Nonsignificant or significant at $P \leq 0.05$ or 0.01 , respectively. 
Table 9. Mean myrosinase activity in crude extracts from rapid-cycling Brassica oleracea grown with different photoperiods ${ }^{z}$.

\begin{tabular}{lcc}
\hline \multirow{2}{*}{$\begin{array}{l}\text { Photoperiod } \\
\text { (h) }\end{array}$} & \multicolumn{2}{c}{ Activity } \\
\cline { 2 - 3 } Leaves & $\begin{array}{c}\text { Activity-FW } \\
(\mathrm{U} / \mathrm{g} \pm \mathrm{SE})\end{array}$ & $\begin{array}{c}\text { Specific activity } \\
(\mathrm{U} / \mathrm{mg} \pm \mathrm{SE})\end{array}$ \\
12 & $25.0 \pm 1.6$ & $16.8 \pm 3.0$ \\
18 & $24.3 \pm 2.2$ & $16.0 \pm 3.8$ \\
24 & $29.6 \pm 2.0$ & $18.5 \pm 3.5$ \\
Linear & $\mathrm{NS}$ & $\mathrm{NS}$ \\
Quadratic & $\mathrm{NS}$ & $\mathrm{NS}$ \\
Stems & & $22.7 \pm 5.7$ \\
12 & $21.1 \pm 2.8$ & $17.5 \pm 4.2$ \\
18 & $18.3 \pm 1.5$ & $18.3 \pm 3.3$ \\
24 & $24.3 \pm 2.8$ & $\mathrm{NS}$ \\
Linear & $\mathrm{NS}$ & $\mathrm{NS}$ \\
Quadratic & $\mathrm{NS}$ & $23.2 \pm 8.7$ \\
Roots & & $34.6 \pm 10.1$ \\
12 & $26.5 \pm 4.6$ & $28.3 \pm 6.1$ \\
18 & $41.7 \pm 5.0$ & $\mathrm{NS}$ \\
24 & $45.3 \pm 5.7$ & $\mathrm{NS}$ \\
Linear & $*$ & $\mathrm{NS}$ \\
Quadratic & &
\end{tabular}

leaves grown at $22^{\circ} \mathrm{C}$. Activity-FW in stems was of a similar magnitude and similarly varied with temperature. Activity-FW in stems grown at 12 and $32{ }^{\circ} \mathrm{C}$ was $86 \%$ and $63 \%$ higher, respectively, than in stems grown at $22{ }^{\circ} \mathrm{C}$. In roots, activity-FW at 12 and $32{ }^{\circ} \mathrm{C}$ was similar in magnitude to that in stems and leaves grown at the same temperatures, but at $22^{\circ} \mathrm{C}$, root activity-FW was significantly elevated compared to that in stems and leaves. In consequence, root activity-FW did not change across temperature regimes. Temperature did not affect specific activity (U/mg protein) in leaves, stems, or roots.

ACTIVITY OF MYROSINASE FROM PLANTS GROWN AT DIFFERENT LEVELS OF PPF. In leaves, activity-FW had a positive quadratic relationship with $P P F$ (Table 8 ). Activity-FW in leaves grown under $P P F$ of 200 and $400 \mu \mathrm{mol} \cdot \mathrm{m}^{-2} \cdot \mathrm{s}^{-1}$ was $43 \%$ and $28 \%$ higher respectively compared to that in leaves grown under 300 $\mu \mathrm{mol} \cdot \mathrm{m}^{-2} \cdot \mathrm{s}^{-1}$. In contrast, activity-FW in roots had a negative quadratic relationship with $P P F$. Activity-FW in roots from plants grown under 200 and $400 \mu \mathrm{mol} \cdot \mathrm{m}^{-2} \cdot \mathrm{s}^{-1}$ was $32 \%$ and $39 \%$ lower, respectively, than in roots from the $300-\mu \mathrm{mol} \cdot \mathrm{m}^{-2} \cdot \mathrm{s}^{-1}$ treatment. Activity-FW in stems was not affected by $P P F$.

Specific activity in leaves decreased with increasing $P P F$. Under $P P F$ of $200 \mu \mathrm{mol} \cdot \mathrm{m}^{-2} \cdot \mathrm{s}^{-1}$, specific activity in leaves was $74 \%$ higher than in leaves grown under $P P F$ of $400 \mu \mathrm{mol} \cdot \mathrm{m}^{-2} \cdot \mathrm{s}^{-1}$. Specific activity in stems and roots did not vary with $P P F$.

ACTIVITY OF MYROSINASE FROM PLANTS GROWN WITH DIFFERENT PHOTOPERIODS. Activity-FW in roots increased with photoperiod (Table 9). Under constant light, activity-FW in roots was $71 \%$ higher than in roots grown with a 12-h photoperiod. Photoperiod did not significantly affect activity-FW in leaves or stems, nor did photoperiod influence specific activity in leaves, stems, or roots.

ACTIVITY OF MYROSINASE FROM PLANTS GROWN UNDER FALL OR SPRING SIMULATION CONDITIONS. Mean activity-FW was $65 \%$ higher in leaves from plants grown in the spring simulation than in the fall simulation experiment (Table 10). Similarly activity-FW was $40 \%$ higher in stems from the spring simulation compared to that in stems from the fall simulation. Root activity-FW in the spring simulation did not differ from that in the fall simulation. Specific
Table 10. Mean myrosinase activity in crude extracts from rapid-cycling Brassica oleracea grown in simulated fall or spring conditions.

\begin{tabular}{lcc}
\hline \multirow{2}{*}{$\begin{array}{l}\text { Simulated } \\
\text { season }\end{array}$} & \multicolumn{2}{c}{ Activity } \\
\cline { 2 - 3 } Leaves & $\begin{array}{c}\text { Activity-FW } \\
(\mathrm{U} / \mathrm{g} \pm \mathrm{SE})\end{array}$ & $\begin{array}{c}\text { Specific activity } \\
(\mathrm{U} / \mathrm{mg} \pm \mathrm{SE})\end{array}$ \\
$\quad$ Fall & $18.9 \pm 3.1$ & $22.4 \pm 4.0$ \\
$\quad$ Spring & $31.2 \pm 2.7$ & $18.6 \pm 2.8$ \\
& $*$ & $\mathrm{NS}$ \\
Stems & & $28.2 \pm 1.6$ \\
$\quad$ Fall & $23.5 \pm 1.8$ & $19.3 \pm 2.0$ \\
$\quad$ Spring & $33.0 \pm 0.4$ & $*$ \\
& $*$ & $51.0 \pm 4.3$ \\
Roots & & $46.9 \pm 4.2$ \\
$\quad$ Fall & $58.6 \pm 5.3$ & $\mathrm{NS}$ \\
$\quad$ Spring & $55.9 \pm 1.4$ & $\mathrm{NS}$
\end{tabular}

ss,"Nonsignificant or significant at $P \leq 0.05$, respectively.

activity in leaves and roots did not differ between plants from the fall simulation and the spring simulation. Specific activity in stems from the fall simulation were $46 \%$ higher than in those from the spring simulation.

\section{Discussion}

GLUCOSINOLATES IN THE TEMPERATURE, PPF, AND PHOTOPERIOD EXPERIMENTS. Total GS concentrations in leaves from plants grown at $22{ }^{\circ} \mathrm{C}$ under constant $P P F$ of $300 \mu \mathrm{mol} \cdot \mathrm{m}^{-2 \cdot} \cdot \mathrm{s}^{-1}$ were similar to those of a previous study in which RCBP $B$. oleracea were grown at $23{ }^{\circ} \mathrm{C}$ (Charron et al., 2001). GE and GNS were not detected in leaves or stems in contrast to an earlier investigation where both GSs were at concentrations $<0.5 \%$ of total GSs in shoots at first open flower (Hill et al., 1988). Since shoots include leaves and stems, the detection of GSs in shoots might have occurred due to the contribution from stem tissue. The presence of GE could have been detected due to the harvest at first open flower rather than just prior to anthesis.

Side-chain modification of GE leads to the synthesis of GR in B. oleracea (Giamoustaris and Mithen, 1996). Significant concentrations of GE in root tissue $(\approx 10 \%$ of total GSs) in all experiments may result from relatively inefficient conversion of GE to GR. Since GR but not GE was found in leaves and stems, these tissues more effectively executed this conversion. From a human health standpoint, it may be desirable to enhance concentrations of GR in Brassica food crops. Since the Gsl-alk locus catalyzes the conversion of GR to GNP and then PRO, a null allele at this locus is preferable (Giamoustaris and Mithen, 1996). Based on the fact that GNP and PRO were the predominant GSs in all experiments, the potential for increasing GR concentrations is significant. A functional allele at the Gsl-elong locus is also required since it catalyzes synthesis of the butyl GS derivatives which are precursors to GR (Magrath et al., 1994). Giamoustaris and Mithen (1996) noted that these goals could be met by markerassisted selection of wild or cultivated Brassicas to introduce the desired alleles or by cloning of the Gsl-elong and Gsl-alk genes from Arabidopsis thaliana. Members of the B. villosa-rupestris complex from Sicily have a non-functional $G s l$-alk allele and may be useful in a program to elevate GR concentrations (Faulkner et al, 1998). Mithen et al. (2003) used this selection strategy and produced broccoli with 80 -times the ability to induce quinone reductase compared to standard commercial broccoli. 
Temperature extremes elevated total and aliphatic GSs in leaves, but did not affect indole GSs. This result is in agreement with Rosa and Rodrigues (1998) who determined that in general, total GS concentrations and the fluctuation of ultradian rhythms increased at high temperatures. Pereira et al. (2002) found that in two cultivars of broccoli sprouts, higher concentrations of total and aliphatic GS were present at 11.3 and $33.1{ }^{\circ} \mathrm{C}$ than at intermediate temperatures. Indole GSs in 'Marathon' were not affected by temperature but indole GSs in 'DeCicco' varied inversely with methylthioalkyl GSs (GE, GI, GR), highlighting the fact that individual cultivars may respond differently to environmental influences.

The inverse relationship of total GSs in leaves with that in roots across different temperatures raises the question of whether there is a redistribution of GSs within the plant in response to the temperature extremes. It is possible that GSs are mobilized from roots to shoots at stress-inducing temperatures as part of a survival strategy.

The negative quadratic relationship of total GSs with temperature in roots was due largely to low concentrations of GNS at both temperature extremes. Gluconasturtiin may have a role in defense against pests. The presence of GNS in host plants or synthetic diets reduced development of the host specialist aphid Brevicoryne brassicae (Cole, 1996). The fact that GNS concentrations varied with temperature is consistent with the observation that abundance of insect pests is often temperature-related (Vasicek et al., 1999). Salicylic acid, a phenolic compound associated with defensive responses to pest attack, may be part of a defense system involving GNS. In leaves of oilseed rape (B. napus L.), GNS concentrations were specifically induced by application of a 10-mM salicylic acid root drench (Kiddle et al., 1994). Salicylic acid or some other defense-related compound may have been involved in temperature-induced variation in concentrations of GNS in RCBP of B. oleracea.

Concentrations of indole GSs were suppressed in stems and roots at lower temperatures. When indole GSs in stems were calculated as a percentage of total GSs, indole GSs were $6 \%$, $8 \%$, and $11 \%$ of total GSs in plants grown at 12,22 , and $32{ }^{\circ} \mathrm{C}$, respectively. Indole GSs in roots were $32 \%, 41 \%$, and $60 \%$ of total GSs in plants grown at 12,22 , and $32{ }^{\circ} \mathrm{C}$, respectively. Indole GSs are known to increase systemically in response to methyl jasmonate, another defensive signaling molecule (Barlett et al., 1999; Bodnaryk, 1994; Doughty et al., 1995). Moreover, different cultivars responded differently. GB and NGB increased in response to methyl jasmonate in 'Bievenu' oilseed rape, but only GB increased in 'Westar' plants exposed to a similar treatment. Although indole GSs are induced by methyl jasmonate, and methyl jasmonate is associated with wound and pest responses, indole GSs are not antifeedants and their adaptive role in plants is unclear (Bodnaryk, 1992).

Although the relationship describing total GS concentrations in leaves in response to temperature had linear and quadratic components, the response of total GSs to $P P F$ had only a linear component. The $P P F$ range used (200 to $400 \mu \mathrm{mol} \cdot \mathrm{m}^{-2} \cdot \mathrm{s}^{-1} ; 400$ $\mu \mathrm{mol} \cdot \mathrm{m}^{-2} \cdot \mathrm{s}^{-1}$ was the upper limit of the growth chamber) may not have been expansive enough to cause light-induced stresses at the lowest or highest levels. The linear response may be indicative of a $P P F$-proportional induction of GS biosynthetic enzymes. In contrast, the higher aliphatic and total GSs at $300 \mu \mathrm{mol} \cdot \mathrm{m}^{-2 \cdot} \cdot \mathrm{s}^{-1}$ compared to those at 200 or $400 \mu \mathrm{mol} \cdot \mathrm{m}^{-2 \cdot} \cdot \mathrm{s}^{-1}$ suggests a nonlinear biosynthetic response to $P P F$ levels.

Although total GSs in stems were quadratically and linearly related with $P P F$, concentrations of the group of aliphatic GSs and many individual GSs were inversely related to concentrations of the group of indole GSs and corresponding individual GSs. This inverse relationship could result from a $P P F$-induced shift in the pool of amino acids that are precursors to aliphatic (alanine, methionine, valine, leucine, or isoleucine) and indole (tryptophan) GSs. If this were the case, it is surprising that leaf tissue, which is more exposed to differences in $P P F$, was not similarly affected.

Concentrations of GNS in roots increased with increasing $P P F$. In a study of watercress (Nasturtium officianale $\mathrm{R}$. Br.), concentrations of phenethyl isothiocyanate, the isothiocyanate derivative of GNS, were increased $60 \%$ when $P P F$ was increased from 265 to $435 \mu \mathrm{mol} \cdot \mathrm{m}^{-2} \cdot \mathrm{s}^{-1}$ during the week before harvest (Palaniswamy et al., 1997). This occurred under an 8-h photoperiod but not under a 12-h photoperiod, suggesting an interaction of $P P F$ with photoperiod.

Variations in root GSs with $P P F$ are interesting because the plant reservoirs were impermeable to light. Although low levels of light may have penetrated the polyurethane plant supports, roots were not directly exposed to different levels of $P P F$ and changes in aliphatic GSs would presumably occur in response to differential transport of GS or GS precursors or to chemical signals from leaf or stem tissue. Several investigators have determined that the profile and concentration of GSs differ in plant organs and that GSs can be mobilized by phloem transport (Brown et al., 2003; Rosa et al., 1997; Chen and Andreassson, 2001). The photoperiodic response of aliphatic and total GSs in roots also requires a chemical signal or transport from shoot tissue since roots were not directly exposed to photoperiodic changes.

GI is a precursor of SN. The absence of GI in leaves or roots grown under a 12-h photoperiod and in roots grown under an 18 -h photoperiod and the presence of SN in these treatments suggests an accelerated conversion of GI to SN. The finding that GI concentrations were influenced by photoperiod is in agreement with Rosa and Rodrigues (1998) who reported that photoperiod affected ultradian rhythms of GI concentrations in cabbage seedlings.

GLUCOSINOLATES IN THE FALL SIMULATION AND SPRING SIMULATION EXPERIMENTS. The fall simulation and spring simulation experiments introduced the confounding effects of simultaneously changing temperature, $P P F$, and photoperiod. It was not possible to grow the plants for the identical number of days at each level in both experiments and have developmentally similar plants at harvest (Table 2). For example, during the final phase of the fall simulation experiment under $16.0^{\circ} \mathrm{C}$ (light temperature), $8.0^{\circ} \mathrm{C}$ (dark temperature), $200 \mu \mathrm{mol} \cdot \mathrm{m}^{-2} \cdot \mathrm{s}^{-1}$, and 12 -h photoperiod, $5 \mathrm{~d}$ were required to grow the plants to the point just before anthesis. Only $2 \mathrm{~d}$ were needed for the final stage of the spring simulation experiment. In addition, it is difficult to know what stage or stages of each experiment were most critical for GS biosynthesis as plants were exposed to changing environmental parameters. These two experiments can best be understood and interpreted in terms of their generally inversely related trends in temperature, $P P F$, and photoperiod.

The results of the season simulation experiment were in agreement with several studies that measured GSs in B. oleracea in different seasons. Total and individual GSs were generally higher in broccoli inflorescences grown as a spring crop compared to a fall crop, although some cultivar differences were observed (Rosa and Rodrigues, 2001). In a study of eight broccoli cultivars, aliphatic and indole GSs were affected by cultivar and season, and 
highest GSs occurred in late season crops (grown from March to April) compared to early season crops [grown from December to February (Vallejo et al., 2003)]. Glucosinolate concentrations in two cultivars of Portuguese cabbage $[B$. oleracea L. (Costata Group)], one Portuguese kale [B. oleracea L. (Acephala Group)], one hybrid white cabbage, and one Portuguese Brassica napus type were higher in spring and summer than in summer and winter (Rosa et al., 1996). These results are in contrast to those of Agerbirk et al. (2001) who measured higher total GSs later in the fall compared to earlier in the fall in leaves of natural populations of Barbarea vulgaris ssp. arcuata. Since the plants were not controlled for age, planting times, or growing conditions, it is impossible to know what factors contributed to the trend of higher GSs later in the fall season.

Aliphatic and total GS concentrations in leaves were higher at $32{ }^{\circ} \mathrm{C}$ than at $12{ }^{\circ} \mathrm{C}$ in the temperature experiment, and lower at $400 \mathrm{~mol} \cdot \mathrm{m}^{-2} \cdot \mathrm{s}^{-1}$ compared to $200 \mu \mathrm{mol} \cdot \mathrm{m}^{-2} \cdot \mathrm{s}^{-1}$ in the $P P F$ experiment. The higher aliphatic and total GSs in leaves of the spring simulation experiment may have resulted from a confluence of these two factors such that the elevating effect of higher temperature was more important than the decreasing effect of higher $P P F$. The final $48 \mathrm{~h}$ of the spring simulation in which plants were grown at $30{ }^{\circ} \mathrm{C}$ under constant $400 \mathrm{~mol} \cdot \mathrm{m}^{-2} \cdot \mathrm{s}^{-1}$ may have been the most important time period in determining GS concentrations at harvest. That temperature was a major influence on total GSs in leaves is supported by the observation that in all experiments, only the 12 and $32{ }^{\circ} \mathrm{C}$ temperature treatments resulted in higher leaf GSs than measured in the spring simulation.

The high concentrations of total GSs in stems from the spring simulation compared to the fall simulation were not characteristic of high temperature or high levels of $P P F$, although total GSs in the 24 -h photoperiod treatment were $43 \%$ higher than in the $12-\mathrm{h}$ photoperiod. Total GSs in stems from the spring simulation were $14 \%$ higher than the next highest value measured in any experiment $\left(32{ }^{\circ} \mathrm{C}\right.$ treatment). Interactive effects of temperature, $P P F$, and photoperiod may account for the fact that high concentrations of GSs in stems from the spring simulation were not found in any experiment in which only one environmental factor was varied.

Myrosinase aCtIVITY IN THE TEMPERATURE, PPF, AND PHOTOPERIOD EXPERIMENTS. There are no reports describing levels of myrosinase activity in RCBP $B$. oleracea. The range of activityFW measured in this study is higher than that found in broccoli sprouts (3.03 U/g FW) although the stages of development (RCBP $B$. oleracea harvested just before anthesis vs. broccoli sprouts harvested after 3 d) were not comparable (Shapiro et al., 2001). Leaves of nasturtium (Tropaeolum majus L.) had activity of 30.6 $\mathrm{U} / \mathrm{g}$ DW (Wielanek and Urbanek, 1999). If it is assumed that nasturtium leaves contain $85 \%$ water, then this activity would be equivalent to 4.6 U/g FW. In contrast, James and Rossiter (1991) recorded a value of $123 \mathrm{U} / \mathrm{g}$ FW in cotyledons of $B$. napus. Specific activity in a variety of Brassica vegetables ranged from 2.0 $\mathrm{U} / \mathrm{mg}$ protein [Chinese kale (B. oleracea L. (Alboglabra Group)] to $22.6 \mathrm{U} / \mathrm{mg}$ protein for radish [(Raphanus sativus $\mathrm{L}$.); Yen and Wei, 1993). Differences in activity measured in RCBP B. oleracea compared to that found by others may result from the use of different plant varieties, developmental stages, and laboratory (extraction, purification, and measurement) techniques.

The elevated activity-FW in leaves and stems grown at 12 and $32{ }^{\circ} \mathrm{C}$ is important because myrosinase is integral to reactions that give Brassica crops their chemoprotective potential. Although the human digestive system has some capacity to metabolize GSs, the presence of plant myrosinase enhances this ability significantly
(Getahun and Chung, 1999; Shapiro et al., 1998). Thus when human nutrition is a primary concern, growing temperature may be an important consideration when timing the planting of cruciferous crops in the field.

The loss of statistical significance with temperature when myrosinase activity was calculated on the basis of protein content means that higher levels of protein were present when higher levels of activity-FW were measured. Such a situation was observed in hypocotyls of Brassica napus; hypocotyls had higher specific activity than cotyledons, but higher total activity and higher protein levels were measured in cotyledons (Bones, 1990). It would be understandable for activity-FW to increase as a result of more myrosinase enzyme, but that is not the only possibility. Protein levels in plants respond to environmental stresses. Heat shock proteins accumulate at high temperatures and cold-acclimation proteins accumulate at low temperatures (Gilmour et al., 1988; Lindquist and Craig, 1988).

Among the $P P F$ treatments, activity-FW and specific activity were highest in leaves grown under $200 \mu \mathrm{mol} \cdot \mathrm{m}^{-2} \cdot \mathrm{s}^{-1}$. Myrosinase activity in hypocotyls of dark-grown Sinapsis alba L. seedlings was higher than in light-grown seedlings (Pihakaski and Pihakaski, 1978). Illumination with blue light induced myrosinase gene expression and myrosinase activity in radish hypocotyls (Hasegawa et al., 2000; Yamada et al., 2003). Light quality did not vary during the $P P F$ experiments since $P P F$ at plant level was selected by adjusting the distance between the plants and the lights. Nonetheless, these studies confirm a role for light in inducing myrosinase activity during plant development.

The increase of activity-FW in roots with photoperiod is another case in which protein synthesis in roots may be triggered by a signal from shoot tissue or myrosinase is transported from shoots to roots. The possibility of myrosinase transport has been suggested before. Chen and Andreasson (2001) noted that the presence of myrosin cells adjacent to GS-rich S-cells in Arabadopsis flower stalk may indicate the transport of myrosinase via plasmodesmata. In another study, blue light-illumination of radish hypocotyl halves resulted in increased myrosinase activity in shaded halves, perhaps caused by a signaling or transport mechanism (Yamada et al., 2003).

Myrosinase aCTIVITY IN THE FALL SIMULATION AND SPRING SIMULATION EXPERIMENTS. Although activity-FW was higher in leaves and stems in the spring simulation than in the fall simulation, specific activity in leaves was not different between simulated seasons and specific activity in stems was higher in the fall simulation. These results reflect higher levels of crude protein in plants grown in the spring simulation compared to the fall simulation. Rao and Horn (1986) found that the crude protein concentration of fall-grown Brassica crops was higher than in spring-grown crops. Protein concentration in Portuguese cabbage was up to $84 \%$ higher in the summer/winter season compared to the spring and summer (Rosa, 1996). In the season simulation experiments, environmental variables changed more rapidly than normally occurs in a field situation. Temperature, for example, increased by $3.5^{\circ} \mathrm{C}$ every 3 to $4 \mathrm{~d}$ during the light period in the spring simulation. Protein synthetic rates in the simulation experiments may not have varied at the same rate as the environmental variables, and the rate of change of myrosinase synthesis with changing environment in the simulation experiments may not have approximated changes occurring in field-grown crops. Such a situation could explain why crude protein levels in the spring simulation were higher than in the fall simulation whereas in the field, fall-grown Brassica crops had higher protein levels than spring-grown crops (Rao and Horn, 1986; Rosa, 1996). 
The response of myrosinase activity to various environmental factors may reflect the induction of different myrosinase isozymes. Several myrosinase isozymes have been reported and optimum myrosinase activity is known to vary by isozyme, organ, and species (Buchwaldt et al., 1986; Kozlowska et al., 1983; Ludikhuyze et al., 2000; Yen and Wei, 1993). Patterns of isozymes can change in response to external influences such as wounding or sulfur deficiency (Bones et al., 1994; Taipalensuu et al., 1997; Visvalingam et al., 1998), and the results of this experiment may reflect differential induction of myrosinase isozymes by temperature, $P P F$, and photoperiod.

\section{Conclusions}

Temperature, $P P F$, and photoperiod influenced GS concentrations and myrosinase activity in leaves, stems, and roots. Temperature and $P P F$ were more instrumental in inducing changes in GSs and myrosinase activity than photoperiod. In the season simulation experiment, the effects of individual environmental factors were not additive, and factors may have functioned interactively in influencing GS concentrations and myrosinase activity. Exploitation of the glucosinolate-myrosinase system to optimize the cancer chemoprotective attributes of Brassica crops should include consideration of climatic factors when deciding planting time and location.

\section{Literature Cited}

Agerbirk, N., C.E. Olsen, and J.K. Nielsen. 2001. Seasonal variation in leaf glucosinolates and insect resistance in two types of Barbarea vulgaris ssp. arcuata. Phytochemistry 58:91-100.

Bartlett, E., G. Kiddle, I. Williams, and R. Wallgrove. 1999. Woundinduced increases in the glucosinolate content of oilseed rape and their effect on subsequent herbiovory by a crucifer specialist. Entomol. Expt. Appl. 91:163-167.

Bodnaryk, R. 1992. Effects of wounding on glucosinolates in the cotyledons of oilseed rape and mustard. Phytochemistry 31:2671-2677.

Bodnaryk, R. 1994. Potent effect of jasmonates on indole glucosinolates in oilseed rape and mustard. Phytochemistry 35:301-305.

Bones, A.M. 1990. Distribution of $\beta$-thioglucosidase activity in intact plants, cell and tissue cultures and regenerant plants of Brassica napus L. J. Expt. Bot. 227:737-744.

Bones, A.M. and T.H. Iversen. 1985. Myrosin cells and myrosinase. Isr. J. Bot. 34:351-375.

Bones, A.M., S. Visvalingam, and O.P. Thangstad. 1994. Sulphate can induce differential expression of thioglucoside glucohydrolases (myrosinases). Planta 193:558-556.

Bradford, M.M. 1976. A rapid and sensitive method for the quantitation of microgram quantities of protein utilizing the principle of protein-dye binding. Anal. Biochem. 72:248-254.

Brown, P.D., J.G. Tokuhisa, M. Reichelt, J. Gershenzon. 2003. Variation of glucosinolate accumulation among different organs and developmental stages of Arabidopsis thaliana. Phytochemistry 62:471-481.

Buchwaldt, L., L.M. Larsen, A. Plöger and H. Sørensen. 1986. Fast polymer liquid chromatography, isolation, and characterization of plant myrosinase, $\beta$-thioglucosidase, isoenzymes. J. Chromatogr. 363:71-80.

Charron, C.S. and C.E. Sams. 2001. Sodium selenate fertilisation increases selenium accumulation and decreases glucosinolate concentration in rapid-cycling Brassica oleracea. J. Sci. Food Agric. 81:962-966.

Chen, S. and E. Andreasson. 2001. Update on glucosinolate metabolism and transport. Plant Physiol. Biochem. 39:743-758.

Ciska, E., B. Martyniak-Przybyszewska, and H. Kozlowska. 2000. Content of glucosinolates in cruciferous vegetables grown at the same site for two years under different climatic conditions. J. Agr. Food Chem. 48:2862-2867.
Cole, R. 1996. Abiotic induction of changes to glucosinolate profiles in Brassica species and increased resistance to the specialist aphid Brevicoryne brassicae. Entomol. Expt. Appl. 80:228-230.

Doughty, K.J., G. Kiddle, B.J. Pye, and R.M. Wallgrove. 1995. Selective induction of glucosinolates in oilseed rape leaves by methyl jasmonate. Phytochemistry 38:347-350.

Fahey, J.W. and P. Talalay. 1999. Antioxidant functions of sulforaphane: a potent inducer of Phase II detoxication enzymes. Food Chem. Toxicol. 37:973-979.

Fahey, J.W., Y. Zhang, and P. Talalay. 1997. Broccoli sprouts: An exceptionally rich source of inducers of enzymes that protect against chemical carcinogens. Proc. Natl. Acad. Sci. 94:10367-10372.

Fahey, J.W., A.T. Zalcmann, and P. Talalay. 2001. The chemical diversity and distribution of glucosinolates and isothiocyanates among plants. Phytochemistry 56:5-51.

Faulkner, K., R. Mithen, and G. Williamson. 1998. Selective increase of the potential anticarcinogen 4-methylsulphinylbutyl glucosinolate in broccoli. Carcinogenesis 19:605-609.

Gamet-Payrastre, L., P. Li, S. Lumeau, G. Cassar, M.A. Dupont, S. Chevolleau, N. Gasc, J. Tulliez, and F. Terce. 2000. Sulforaphane, a naturally occurring isothiocyanate, induces cell cycle arrest and apoptosis in HT29 human colon cancer cells. Cancer Res. 60:1426-1433.

Getahun, S.M. and F.L. Chung. 1999. Conversion of glucosinolates to isothiocyanates in humans after ingestion of cooked watercress. Cancer Epidemiol. Biomarkers \& Prevention 8:447-451.

Giamoustaris, A. and R. Mithen. 1996. Genetics of aliphatic glucosinolates. IV. Side-chain modification in Brassica oleracea. Theor. Appl. Genet. 93:1006-1010.

Gilmour, S.J., R.K. Hajela, and M.F. Thomashow. 1988. Cold acclimation in Arabidopsis thaliana. Plant Physiol. 87:745-750.

Hansen, M., P. Møller, H. Sørensen, and M. Cantwell de Trejo. 1995. Glucosinolates in broccoli stored under controlled atmosphere. J. Amer. Soc. Hort. Sci. 120:1069-1074.

Hasegawa, T., K. Yamada, S. Kosemura, S. Yamamura, and K. Hawegawa. 2000. Phototropic stimulation induces the conversion of glucosinolate to phototropism-regulating substances of radish hypocotyls. Phytochemistry 54:275-279.

Hill, C.B., D.G. Carlson, and P.H. Williams. 1988. Glucosinolates in rapid-cycling Brassicas. Cruciferae Nwsltt. 13:50-51.

James, D.C. and J.T. Rossiter. 1991. Development and characteristics of myrosinase in Brassica napus during early seedling growth. Physiol. Plantarum 82:163-170.

Kiddle, G.A., K.J. Doughty, and R.M. Wallsgrove. 1994. Salicylic acidinduced accumulation of glucosinolates in oilseed rape (Brassica napus L.) leaves. J. Expt. Bot. 45:1343-1346.

Kozlowska, H.J., H. Nowak, and J. Nowak. 1983. Characterization of myrosinase in Polish varieties of rapeseed. J. Sci. Food Agr. 34: 1171-1178.

Kushad, M.M., A.F. Brown, A.C. Kurilicy, J.A. Juvik, B.P. Klein, M.A.Wallig, and E.H. Jeffery. 1999. Variation of glucosinolates in vegetable crops of Brassica oleracea. J. Agr. Food Chem. 47: 1541-1548.

Li, Q., S.D. Eigenbrode, G.R. Stringam, and M.R. Thiagarajah. 2000. Feeding and growth of Plutella xylostella and Spodoptera eridania on Brassica juncea with varying glucosinolate concentrations and myrosinase activities. J. Chem. Ecol. 26:2401-2419.

Lindquist, S. and E.A. Craig. 1988. The heat-shock proteins. Annu. Rev. Gen. 22:631-677.

London, S.J., J.M. Yuan, F.C. Chung, Y.T. Gao, G.A. Coetzee, R.K. Ross, and M.C. Yu. 2000. Isothiocyanates, glutathione S-transferase M1 and T1 polymorphism, and lung-cancer risk: A prospective study of men in Shanghai, China. Lancet 356:724-729.

Ludikhuyze, L., L. Rodrigo, and M. Hendrickx. 2000. The activity of myrosinase from broccoli (Brassica oleracea L. cv. Italica): Influence of intrinsic and extrinsic factors. J. Food Prot. 63:400-403.

Magrath R., M. Morgen, F. Bano, I. Parkin, A. Sharpe, C. Lister, J. Turner, C. Dean, D. Lydiate, and R. Mithen. 1994. Genetics of aliphatic glucosinolates I. Side-chain elongation in Brassica napus and 
Arabidopsis thaliana. Heredity 72:290-299.

Michaud, D.S., D. Spiegelman, S.K. Clinton, E.B. Rimm, W.C. Willett, and E.L. Giovannucci. 1999. Fruit and vegetable intake and incidence of bladder cancer in a male prospective cohort. J. Nat. Cancer Inst. 91:605-613.

Mitchell-Olds, T., D. Siemens, and D. Pedersen. 1996. Physiology and costs of resistance to herbivory and disease in Brassica. Entomol. Expt. Appl. 80:231-237.

Mithen, R., K. Faulkner, R. Magrath, P. Rose, G. Williamson, and J. Marquez. 2003. Development of isothiocyanate-enriched broccoli, and its enhanced ability to induce phase 2 detoxification enzymes in mammalian cells. Theor. Appl. Genet. 106:727-734.

Palaniswamy, U., R. McAvoy, and B. Bible. 1997. Supplemental light before harvest increases phenethyl isothiocyanate in watercress under 8-hour photoperiod. HortScience 32:222-223.

Palmieri, S., O. Leoni, and R. Iori. 1982. A steady-state kinetics study of myrosinase with direct ultraviolet spectrophotometric assay. Anal. Biochem. 123:320-324.

Pereira, F.M.V., E. Rosa, J.W. Fahey, K.K. Stephenson, R. Carvalho, and A. Aires. 2002. Influence of temperature and ontogeny on the levels of glucosinolates in broccoli (Brassica oleracea var. italica) sprouts and their effect on the induction of mammalian phase 2 enzymes. J. Agr. Food Chem. 50:6239-6244.

Pihakaski, S. and K. Pihakaski. 1978. Myrosinase in Brassicaceae (Cruciferae). III. Effect of ascorbic acid on myrosinases from Sinapsis alba L. seedlings. J. Expt. Bot. 29:1363-1369.

Raney, J.P. and D.I. McGregor. 1990. Determination of glucosinolate content by gas liquid chromatography of trimethylsilyl derivatives of desulfated glucosinolates. Proc. Oil Crops Network, Shanghai, China, 21-23 Apr.

Rao, S.C. and F.P. Horn. 1986. Planting season and harvest date effects on dry matter production and nutrition value of Brassica spp. in the Southern Great Plains. Agron. J. 78:327-333.

Rosa, E. 1996. The influence of growing season on protein and mineral content of several Brassica crops, p. 261-268. In: J.S. Dias, I. Crute, and A.A. Monteiro (eds.). ISHS Acta Horticulturae, ISHS Brassica Symposium. Lisbon Portugal, Vol. 407.

Rosa, E.A.S. 1997. Daily variation in glucosinolate concentrations in the leaves and roots of cabbage seedlings in two constant temperature regimes. J. Sci. Food Agric. 73:364-368.

Rosa, E.A.S., R.K. Heaney, G.R. Fenwick and C.A.M. Portas. 1997. Glucosinolates in crop plants, p. 99-215. In: J. Janick, (ed.). Horticultural reviews, John Wiley \& Sons, New York.

Rosa,E.A.S., R.K.Heaney,C.A.M.Portas, and G.R.Fenwick. 1996. Changes in glucosinolate concentrations in Brassica crops (B.oleracea and B.napus) throughout growing seasons. J. Sci. Food Agr. 71:237-244.

Rosa, E.A.S. and A.S. Rodrigues. 1998. The effect of light and temperature on glucosinolate concentration in the leaves and roots of cabbage seedlings. J. Sci. Food Agr. 78:208-212.

Rosa, E.A.S. and A.S. Rodrigues. 2001. Total and individual glucosinolate content in 11 broccoli cultivars grown in early and late seasons. Hortscience 36:56-59.
Sang, J.P., C.A. Bluett, B.R. Elliott, and J.W. Truscott. 1986. Effect of time of sowing on oil content, erucic acid and glucosinolate contents in rapeseed (Brassica napus L. cv. Marnoo). Austral. J. Expt. Agr. 26:607-611.

Sarwar, M. and J.A. Kirkegaard. 1998. Biofumigation potential of brassicas. II. Effects of environment and ontogeny on glucosinolate production and implications for screening. Plant and Soil 201:91-101.

SAS Institute. 1996. SAS/STAT user's guide, version 6, SAS Inst., Cary, N.C.

Shapiro, T.A., J.W. Fahey, K.L. Wade, K.K. Stephenson, and P. Talalay. 1998. Human metabolism and excretion of cancer chemoprotective glucosinolates and isothiocyanates of cruciferous vegetables. Cancer Epidemiol. Biomarkers \& Prevention 7:1091-1100.

Shapiro, T.A., J.W. Fahey, K.L. Wade, K.K. Stephenson, and P. Talalay. 2001. Chemoprotective glucosinolates and isothiocyanates of broccoli sprouts: Metabolism and excretion in humans. Cancer Epidemiol. Biomarkers Prev. 10:501-508.

Talalay, P. 1999. The war against cancer: New hope. Proc. Amer. Phil. Soc:143:51-72.

Taipalensuu, J.,A. Falk, and L. Rask. 1997. Myrosinase-binding proteins are derived from a large wound-inducible and repetitive transcript. Eur. J. Biochem. 243:605-611.

Vallejo, F., F.A. Tomás-Barberán, A. Gonzalez Benavente-García, and C. García-Viguera. 2003. Total and individual glucosinolate contents in inflorescences of eight broccoli cultivars grown under various climatic and fertilisation conditions. J. Sci. Food Agr. 83:307-313.

Vasicek, A.L., F.R. La Rossa, and S.A. Ramos. 1999. Host and temperature effect on the cabbage aphid, Brevicoryne brassicae L. (Homoptera: Aphidoidea). Horticultura Argentina 18:44-45.

Visvalingam, S., T.G. Hønsi, and A.M. Bones. 1998. Sulphate and micronutrients can modulate the expression levels of myrosinases in Sinapsis alba plants. Physiol. Plant. 104:30-37.

Wielanek, M., and H. Urbanek. 1999. Glucotropaeolin and myrosinase production in hairy root cultures of Tropaeolum majus. Plant Cell Tiss. Org. Cult. 57:39-45.

Yamada, K., T. Hasegawa, E. Minami, N. Shibuya, S. Kosemura, S. Yamamura, and K.I. Hasegawa. 2003. Induction of myrosinase gene expression and myrosinase activity in radish hypocotyls by phototropic stimulation. J. Plant Physiol. 160:255-259.

Yang, C.S., T.J. Smith, and J. Hong. 1994. Cytochrome P-450 enzymes as targets for chemoprevention against chemical carcinogenesis and toxicity: Opportunities and limitations. Cancer Res. (Suppl.)54: 1982s-1986s.

Yen, G.C. and Q.K. Wei. 1993. Myrosinase activity and total glucosinolate content of cruciferous vegetables, and some properties of cabbage myrosinase in Taiwan. J. Sci Food Agr. 61:471-475.

Zhang, Y., T.W. Kensler, C.G. Cho, G.H.Posner, and P. Talalay. 1994.Anticarcinogenic activities of sulforaphane and structurally related synthetic norbornyl isothiocyanates. Proc. Natl. Acad. Sci. 91:3147-3150.

Zhang, Y., P. Talalay, C.G. Cho, and G.H. Posner. 1992. A major inducer of anticarcinogenic protective enzymes from broccoli: Isolation and elucidation of structure. Proc. Natl. Acad. Sci. U.S.A. 89:2399-2403. 